\title{
DMAP: a connectivity map database to enable identification of novel drug repositioning candidates
}

\author{
Hui Huang ${ }^{1}$, Thanh Nguyen ${ }^{2}$, Sara Ibrahim³ ${ }^{3}$ Sandeep Shantharam ${ }^{1}$, Zongliang Yue ${ }^{1}$, Jake Y Chen ${ }^{4,1,2^{*}}$ \\ From 12th Annual MCBIOS Conference \\ Little Rock, AR, USA. 13-14 March 2015
}

\begin{abstract}
Background: Drug repositioning is a cost-efficient and time-saving process to drug development compared to traditional techniques. A systematic method to drug repositioning is to identify candidate drug's gene expression profiles on target disease models and determine how similar these profiles are to approved drugs. Databases such as the CMAP have been developed recently to help with systematic drug repositioning.
\end{abstract}

Methods: To overcome the limitation of connectivity maps on data coverage, we constructed a comprehensive in silico drug-protein connectivity map called DMAP, which contains directed drug-to-protein effects and effect scores. The drug-to-protein effect scores are compiled from all database entries between the drug and protein have been previously observed and provide a confidence measure on the quality of such drug-to-protein effects.

Results: In DMAP, we have compiled the direct effects between 24,121 PubChem Compound ID (CID), which were mapped from 289,571 chemical entities recognized from public literature, and 5,196 reviewed Uniprot proteins. DMAP compiles a total of 438,004 chemical-to-protein effect relationships. Compared to CMAP, DMAP shows an increase of 221 folds in the number of chemicals and 1.92 fold in the number of ATC codes. Furthermore, by overlapping DMAP chemicals with the approved drugs with known indications from the TTD database and literature, we obtained 982 drugs and 622 diseases; meanwhile, we only obtained 394 drugs with known indication from CMAP. To validate the feasibility of applying new DMAP for systematic drug repositioning, we compared the performance of DMAP and the well-known CMAP database on two popular computational techniques: drug-drugsimilarity-based method with leave-one-out validation and Kolmogorov-Smirnov scoring based method. In drugdrug-similarity-based method, the drug repositioning prediction using DMAP achieved an Area-Under-Curve (AUC) score of 0.82, compared with that using CMAP, AUC $=0.64$. For Kolmogorov-Smirnov scoring based method, with DMAP, we were able to retrieve several drug indications which could not be retrieved using CMAP. DMAP data can be queried using the existing C2MAP server or downloaded freely at: http://bio.informatics.iupui.edu/cmaps

Conclusions: Reliable measurements of how drug affect disease-related proteins are critical to ongoing drug development in the genome medicine era. We demonstrated that DMAP can help drug development professionals assess drug-to-protein relationship data and improve chances of success for systematic drug repositioning efforts.

\footnotetext{
* Correspondence: jakechen@iupui.edu

${ }^{4}$ Institute of Biopharmaceutical Informatics and Technology, Wenzhou

Medical University, Zhejiang, China

Full list of author information is available at the end of the article
}

(0) 2015 Huang et al. This is an Open Access article distributed under the terms of the Creative Commons Attribution License (http:// creativecommons.org/licenses/by/4.0), which permits unrestricted use, distribution, and reproduction in any medium, provided the original work is properly cited. The Creative Commons Public Domain Dedication waiver (http://creativecommons.org/publicdomain/ zero/1.0/) applies to the data made available in this article, unless otherwise stated. 


\section{Background}

To reposition drugs [1-3] from one approved indication to a new indication, drug developers could significantly save associated development cost [4] and lower development risks[5]. With the rapid accumulation of genomics, functional genomics, and chemical informatics data in the past decade, several new systematic approaches to drug repositioning have been proposed. For example, one may study the drug-ligand structural binding relationships systematically for all approved drugs to discover their new targets implicated in other diseases using chemoinformatic tools [6]. If the drug-drug similarity relationships, disease-disease similarity relationships, or side-effect-to-side-effect similarity relationships [7] are characterized, one may populate indications from one drug to another among all drugs under study that are closely related through shared disease, shared side effect, or shared target relationship profiles. Machine learning [1] and biomedical literature text mining [8] approaches can also help uncover non-obvious relationships between approved drugs and potential new indications.

Recently, there has been surging interest to apply "connectivity map" (CMAP) techniques, which attempt to match a repositioned drug's effects by their shared disease perturbation gene expression profiles [2,3,9-11]. A major resource-CMAP-was developed by Lamb et al. [11] to assay genome-wide transcriptional expression data across a wide range of cell lines treated with small drug molecules. Based on the CMAP data, Iorio et al. [3] proposed a drug repositioning method by constructing drug-drug similarity networks. Hu and Agarwal[9] and Sirota et al. [2] also investigated how to pair drugs and disease indications based on negative correlation of drug perturbation and disease gene expression patterns identified from CMAP. The anti-correlation relationships between the drugs and diseases are demonstrated to suggest novel therapeutic indications for existing drugs. The primary advantage of CMAP is that it does not require prior knowledge of drug targets or a drug's detailed mechanism of actions to work. However, CMAP's limitation is also quite apparent: limited coverage of drugs, limited drug perturbation gene expression data, limited dosage-dependent conditions, and the dubious transferability of expression patterns from cell lines or animal models to human systems. Ultimately, it can be time-consuming and costly before a significant portion of current drugs in all safe dosage conditions can be tested in even a limited number of cell lines for CMAP according to the statistics in [12].

In this work, we describe our development of a new resource called DMAP that can help drug development researchers evaluate what effects a drug may have on disease-relevant genes or proteins. DMAP compiles each drug's stimulatory or inhibitory effects on genes or their protein products (Figure 1), based on the computational integration of such data from different databases. It covers 438,004 chemical-to-protein effect relationships between 24,121 PubChem compounds that cover 289,571 chemical entities with a synonymous name, and 5,196 distinct UniProt proteins. DMAP may be used wherever CMAP data coverage is poor for drug repositioning applications. To evaluate the DMAP performances, we calculated drug-to-drug similarity based on newly generated DMAP profiles [3] and obtained Kolmogorov-Smirnov test scores $[2,11]$. We demonstrate that DMAP can successfully recall known drugs for examined disease indications. In addition, by applying

DMAP (i.e. Drug directionality Map) construction and evaluation

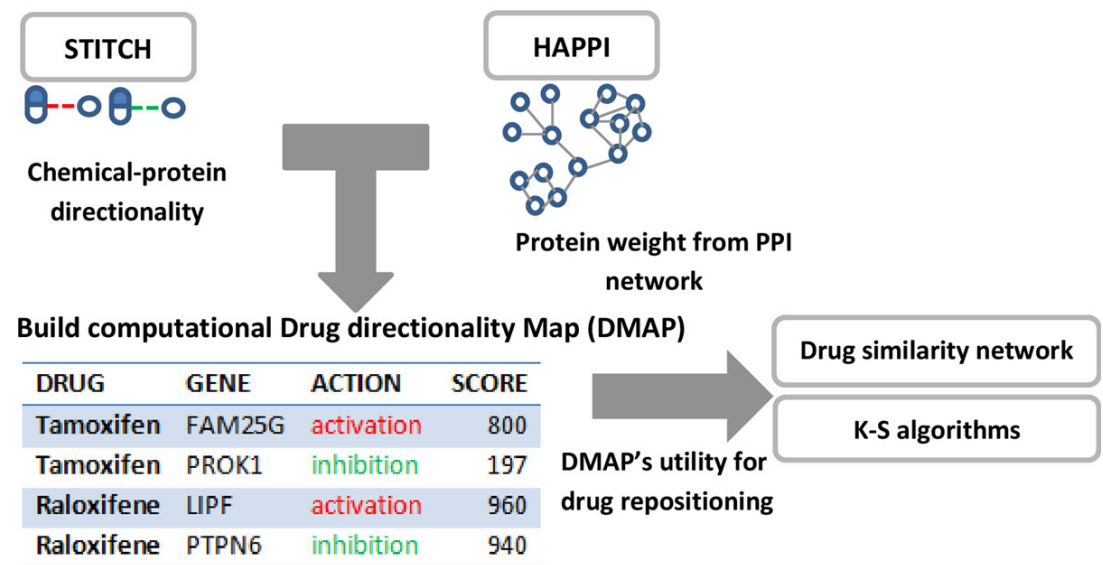

Figure 1 Computational framework. Construct the Drug directionality Map (DMAP) dataset from STITCH and HAPPI and evaluate DMAP's utility for drug repositioning with drug similarity network and K-S algorithms. 
DMAP, we propose novel indications for drugs currently in NCATS [13].

\section{Methods}

\section{Develop DMAP context from existing databases}

In DMAP, we collected, integrated and ranked each pair of drug-to-protein/gene relationship. The primary data for drug-protein information comes from the STITCH [14] database, and may be expanded easily to include other sources such as CTD [15] data. STITCH is an aggregated Cheminformatics database of chemical-to-protein interactions connecting over 300,000 chemicals and 2.6 million proteins for many species mined from biomedical literature. We parsed out STICH chemical protein interactions for Homo sapiens with those chemical-protein "edge actions" being either "activation" (stimulatory interaction) or "inhibition" (inhibitory interactions). To eliminate the synonymous chemicals with the same chemical structure, we mapped 289,571 chemicals to the PubChem database in the result of 24,121 distinct PubChem Compound ID (CID).

Next, we calculated a probability-weighted summary of all the evidence to determine an overall mechanism of "edge action" for each specific chemical-protein interaction using $\operatorname{conf}(d, p)$.

$$
\operatorname{conf}(\mathrm{d}, \mathrm{p})=\sum_{i=1}^{i=N}\left(\operatorname{prob}_{i}(d, p) * \operatorname{sign}_{i}\right)
$$

where $d$ and $p$ are specific drugs and proteins, respectively. $N$ is the number of evidence for the interaction between $d$ and $p . \operatorname{prob}_{i}(d, p)$ is the confidence of each evidence $i$ with a value within the range of $0[1]$. $s i g n_{i}$ has a value of 1 if the evidence $i$ represents activation while has a value of -1 if the evidence $i$ represents inhibition.

Then, to rank each interaction, we used HAPPI [16], an integrated protein interaction database that comprehensively integrated weighted human protein-protein interaction data from HPRD, BIND, MINT, STRING, and OPHID by assigning a weight weight $(p)$ for each drug's interacting proteins using the following formula adapted from [17].

$$
\operatorname{weight}(p)=k \times \ln \left(\sum_{q \in N E T} \operatorname{conf}(p, q)\right)-\ln \left(\sum_{q \in N E T} N(p, q)\right)
$$

Here, $p$ and $q$ are proteins on the protein interaction network, $k$ is an empirical constant ( $k=2$ in this study), $\operatorname{conf}(p, q)$ is the confidence score assigned by HAPPI to each interaction between protein $p$ and $q$, and $N(p, q)$ holds the value of 1 if protein $\mathrm{p}$ interacts with $\mathrm{q}$ or the value of 0 if protein $\mathrm{p}$ does not interact with $\mathrm{q}$.

Finally, we developed an intuitive pharmacology score (P-Score) to combine the probability for each interaction and the weight of the interacting proteins:

$$
P-\operatorname{Score}(d, p)=\operatorname{conf}(d, p) \times \operatorname{weight}(p)
$$

Here, P-Score contains both the information of each drug's action on their interacting proteins and the importance of the protein in the protein-protein interaction network. This is different than the expression level based ranking in CMAP, which may be more suitable for biomarker discovery instead of drug discovery. With P-Score for each drug-protein interaction, DMAP is thus in a compatible format with CMAP [11].

\section{Integrate drug therapeutic indication data}

To construct a golden standard of known drug indications to evaluate DMAP's drug repositioning performance, we integrated the Therapeutic Target Database (TTD) [18] and the dataset from the PREDICT [1] paper. TTD is a database that provides information about drugs' known therapeutic protein targets and their targeted diseases. The PREDICT paper provides a compiled list of drug indications. We integrated these two sources to get 2,912 drug indication associations corresponding to 1,180 drugs and 726 indications.

\section{Prepare disease expression signatures and drug expression signatures}

To apply the Kolmogorov-Smirnov algorithms with DMAP or CMAP for the drug repositioning, we need the disease expression dataset as one of the inputs. We thus retrieved the disease gene expression profiles from Pacini C et al. [19]'s paper. In total, 87 disease associated microarray experiments were compiled to represent 45 distinct diseases. According to Pacini C's paper, these datasets were obtained from the GEO microarray repository [20]. The raw CEL files were normalized with RMA [21]. For those gene expression profiles representing the same disease, they were combined with the median rank normalization by Warnat et al. [22].

The drug-gene expression datasets were obtained from Iorio et al.[3]'s paper instead of directly from CMAP [11] to reduce the batch effect. Iorio et al.[3] computed a single synthetic ranked list of genes, called Prototype Ranked List (PRL), by merging all the ranked list of the same compound in CMAP. Only consistently overexpressed/underexpressed genes are placed at the top/bottom of the RPL. This helped capture a consensus transcriptional response for each drug. We thus chose to use the PRL to represent the drug signatures from CMAP in this study.

\section{Design drug similarity measurement}

The hypothesis for the drug similarity network approach is: if two drugs were similar, the disease indication for one drug could be potentially assigned to the other drug. To measure the similarity among each drug pair, we computed $\operatorname{SIM}\left(d_{x}, d_{y}\right)$ based on the Tanimoto 
Coefficient between their interacting proteins (4).

$$
\operatorname{SIM}\left(\mathrm{d}_{\mathrm{x}}, \mathrm{d}_{\mathrm{y}}\right)=\frac{\left|\mathrm{p}_{\mathrm{x}+} \cap \mathrm{p}_{\mathrm{y}+}\right|-\left|\mathrm{p}_{\mathrm{x}-} \cap \mathrm{p}_{\mathrm{y}-}\right|}{\left|\mathrm{p}_{\mathrm{x}} \cup \mathrm{p}_{\mathrm{y}}\right|}
$$

Here, $d_{x}$ and $d_{y}$ represent the two specific drugs, $p_{x}$ represents the set of proteins interacting with $d_{x}, p_{y}$ represents the set protein interacting with $d_{y} .\left|p_{x} \cup p_{y}\right|$ is the number of total distinct proteins in $p_{x}$ and $p_{y} . \mid p_{x}$ $+\cap p_{y+} \mid$ is the number of overlapped proteins on which both drugs have identical interactions (i.e. both activate or inhibit the shared proteins). $\left|p_{x-} \cap p_{y-}\right|$ is the number of shared related proteins on which the two drugs have opposite interactions (i.e. one activates while the other inhibits the shared proteins). $\operatorname{SIM}\left(d_{x}, d_{y}\right)$ lies in the range of $[-1,1]$ with 1 representing that the two drugs share the same interacting proteins and the drugs' action on each protein is the same while -1 representing that the two drugs share the same proteins but the drugs' action on each protein is opposite.

\section{Evaluate the prediction performance}

To assess the prediction performance, we implemented the 'Guilt by Association' (GBA) concepts (Figure 2.) presented by Chiang et al[23] and conducted "Leave-One-Out" crossvalidation. For each drug, we removed its known indications and attempted to recover them by considering the indications for its top $\mathrm{N}$ similar drugs found. We calculated overall sensitivity and specificity by varying $\mathrm{N}$-the number

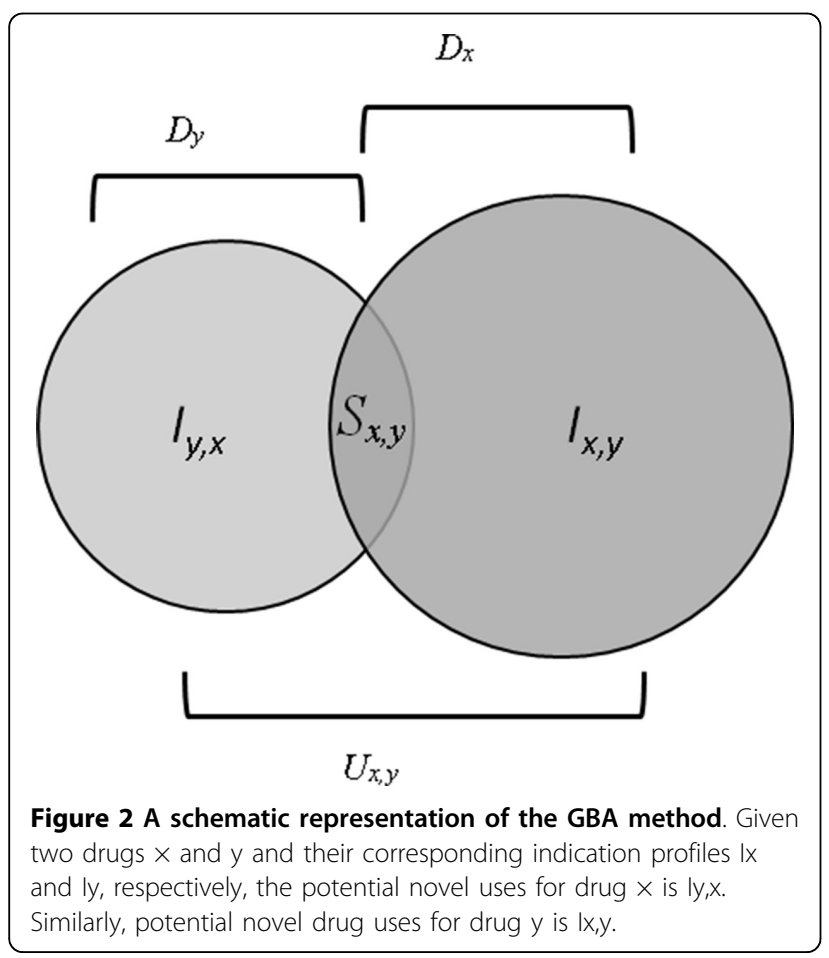

of similar drugs-from 1 to 981 . The area under the ROC curve (AUC) score was used to measure the performance.

\section{Implement Kolmogorov-Smirnov strategy}

We implemented the nonparametric, rank-based strategy based on the algorithm originally introduced by Lamb et al.[11] to generate a ranked list of candidate drugs for each disease. For each disease signature, we computed an enrichment score separately for the up- or down- regulated genes: $\mathrm{es}_{\mathrm{up}}$ and $\mathrm{es}_{\mathrm{down}}$. In specific, we constructed a vector $\mathrm{V}$ of the position of each of the up- or down- regulated genes on the basis of the values from the reference drug dataset. The vector was then sorted in ascending order such that $\mathrm{V}(j)$ is the position of disease gene $j$. The computation of the enrichment score is based on Kolmogorov-Smirnov statistic and the details can be referred to in the supplementary material in Lamb et al. [11]. The drug score is set to zero, where $\mathrm{es}_{\mathrm{up}}$ and $\mathrm{es}_{\text {down }}$ have the same algebraic sign. Otherwise, we set the drug score to $\mathrm{es}_{\mathrm{up}}-\mathrm{es}_{\mathrm{down}}$. To evaluate the statistical significance of the score, we applied a permutation approach by randomly selecting any drug signatures and re-calculated the score accordingly. We did the permutation 200 times for each drug-disease pair and computed the $p$-value by checking the actual score with the score distribution after randomization. We hypothesized that those drugs with a statistically significant negative score might be a possible treatment for the disease of interest.

\section{Perform literature validation}

To check whether the predicted drug-disease pairs have clinical literature evidence, we used the esearch API provided by NCBI. The query term we used is 'drug name AND disease name AND (Clinical Trial[ptyp] OR Clinical Trial, Phase I[ptyp] OR Clinical Trial, Phase II [ptyp] OR Clinical Trial, Phase III[ptyp] OR Clinical Trial, Phase IV[ptyp])'. We recorded the total number of clinical type PubMed articles for each association.

\section{Results and discussion}

\section{Drug directionality Map (DMAP) Construction}

We constructed DMAP containing 438,004 chemical protein interactions for 24,121 PubChem Compound. (Table 1). Compare to CMAP [11], DMAP shows a 14-fold increase of CID coverage. In addition, DMAP cover most of the Anatomical Therapeutic Chemical (ATC) categories: $100 \%$ at the first level, $94.3 \%$ at the second level and $92 . \%$ at the third level. This fact is significant if we compare CMAP coverage on ATC categories: $100 \%$ at the first level, $12.5 \%$ at the second level and $11.7 \%$ at the third level. Comparing to all the drugs from DrugBank, we have $71.5 \%$ in approved group and $12.7 \%$ in experimental group in DMAP that exceeding 
Table 1 Database statistics comparing CMAP (build 02) and the new DMAP.

\begin{tabular}{|c|c|c|}
\hline Count & $\begin{array}{l}\text { CMAP (Build } \\
02 \text { ) }\end{array}$ & DMAP \\
\hline $\begin{array}{l}\text { Chemical entities (including brand } \\
\text { names) }\end{array}$ & 1,309 & 289,571 \\
\hline Drugs with known indications & 394 & 982 \\
\hline $\begin{array}{l}\text { Drug entities with unique PubChem } \\
\text { CID }\end{array}$ & 1,714 & 24,121 \\
\hline Drugbank Approved (and \%) & $569(32.3 \%)$ & $\begin{array}{r}1260 \\
(71.5 \%)\end{array}$ \\
\hline Drugbank Experimental (and \%) & $51(1.0 \%)$ & $646(12.7 \%)$ \\
\hline Coverage of Drug's Therapeutic Areas & 881 & 1,700 \\
\hline ATC first level categories (and \%) & $14(100 \%)$ & $14(100 \%)$ \\
\hline ATC second level categories (and \%) & $72(81.8 \%)$ & $83(94.3 \%)$ \\
\hline ATC third level categories (and \%) & $152(76.7 \%)$ & $184(92.9 \%)$ \\
\hline Proteins by UniProtID (and \%) & $11,820(58.5 \%)$ & $\begin{array}{l}5,196 \\
(25.7 \%)\end{array}$ \\
\hline Drug-to-protein effect relationships & $20,242,271$ & 438,004 \\
\hline Stimulatory effects & $10,156,011$ & 200,310 \\
\hline Inhibitory effects & $10,086,260$ & 237,694 \\
\hline
\end{tabular}

the $39.2 \%$ and $11.7 \%$ respectively in CMAP. The protein and drug-protein interaction coverage in DMAP does not match to CMAP due to CMAP is based on the whole protein screening. The DMAP most popular ATC category distribution is general balance with the DrugBank approved drug distribution except for infectious disease and cancer drugs in category J and L in Figure 3. The reason for the approved drugs in Drugbank little higher rate than DMAP in J and L is probably due to the favor of infectious disease and cancer drug discovery in Drugbank. We also normalize the score range $[-1,1]$ between CMAP and DMAP to perform Pearson correlation and the result is 0.017 , which indicates the difference exists between the cell line drug-protein result, and the protein-chemical

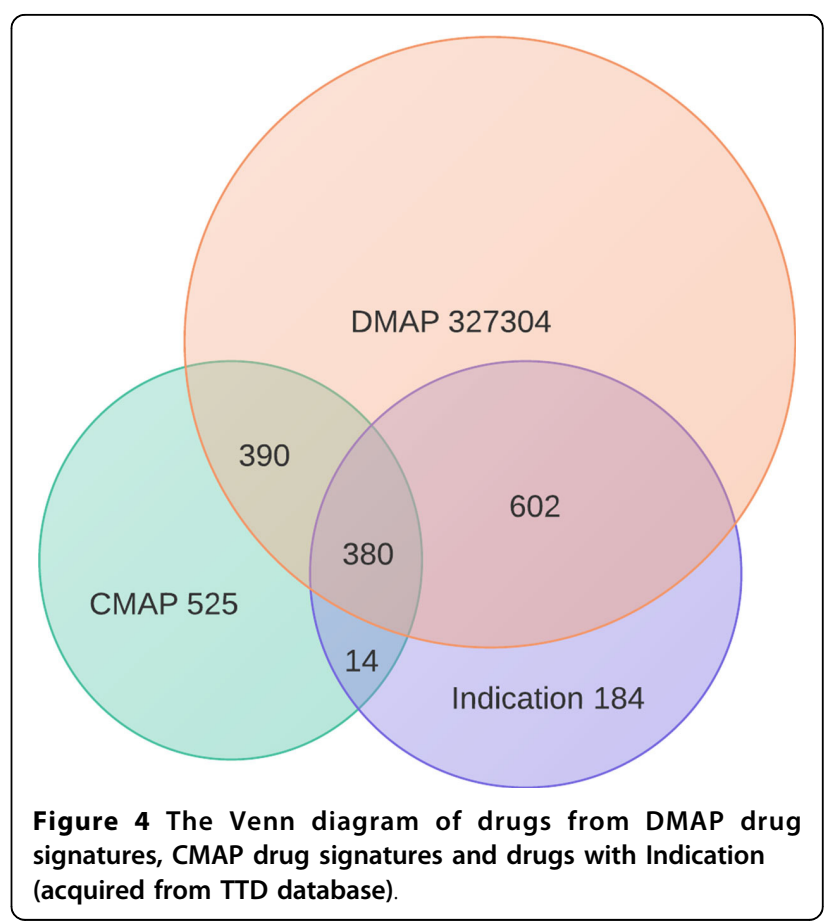

association and predicted drug-downstream target association in human. Figure 4 shows the number of shared chemicals between DMAP, CMAP, and for drugs with known indications which we compiled from the TTD database[24] and literature [1] (Figure 4). CMAP contains 394 drugs with known indications. Meanwhile, DMAP contains 982 drugs with known indication. Among these, CMAP and DMAP share 380 drugs, which cover $96.5 \%$ of CMAP but only $38.70 \%$ of DMAP. CMAP only contains 14 drugs not covered in DMAP; meanwhile DMAP contains 602 drugs not covered in CMAP. Thus, we argue that DMAP provides a valuable resource for repositioning existing drug for new uses. To demonstrate this, in the following section we applied two representative drug-repositioning methods

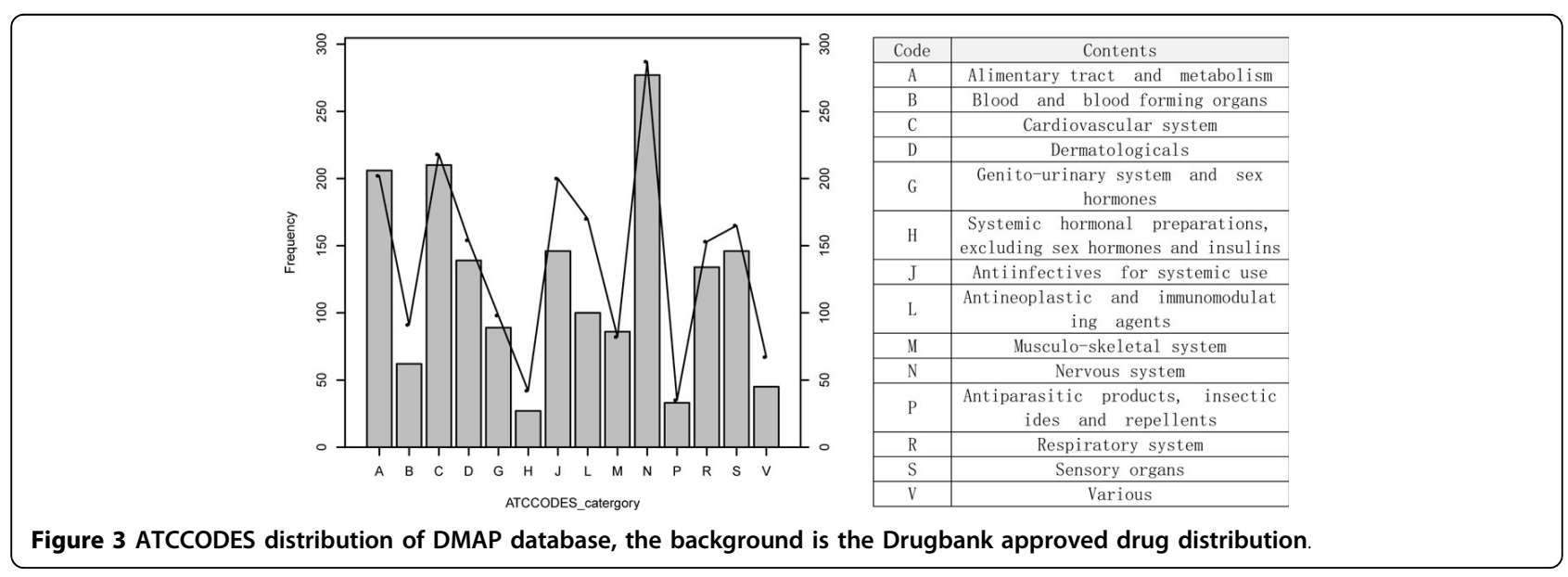



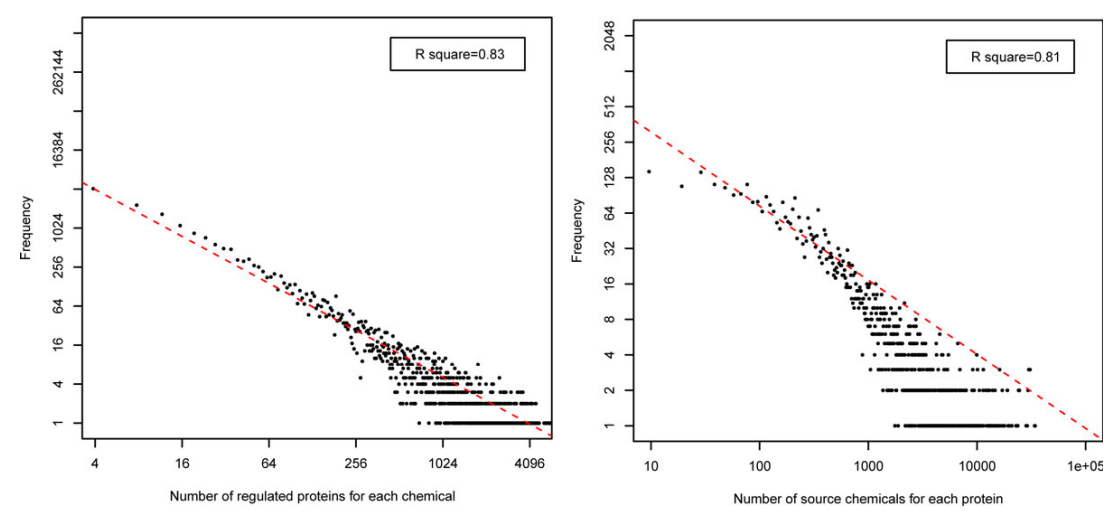

Figure 5 Distribution of protein degrees (A) and drug degrees (B) in DMAP bipartite network.

with DMAP dataset and proved its utility for computational drug repositioning.

In Figure 5, we show the scale-free characteristics of the drug-protein interaction bipartite network in DMAP. Here, the drug degree of a drug is defined as the number of proteins interacting with the drug, and the protein degree of a protein is defined as the number of drugs interacting with the protein. The R-square for linear regression in drug degree and protein degree are 0.83 and 0.81 (in log scale), correspondingly.

Figure 6 shows the gene ontology (GO) terminologies overrepresented (Figure 6A) and underrepresented (Figure 6B) by protein covered in DMAP. Here, we use the FDR calculated by DAVID functional annotation tool [25] on GO to sort the GO terms, and use protein not covered in DMAP to construct the underrepresented GO terms. We observe that GO 'respond' and 'regulation' terms are the most represented in DMAP.

\section{DMAP's utility for drug repositioning}

To check DMAP's utility for drug repositioning, we applied the following two well-known drug-repositioning methods in literatures: (i) drug similarity approach [3], (ii) Kolmogorov-Smirnov algorithms [11].

\section{DMAP outperforms CMAP in repurposing using drug similarity approach}

We computed 481,671 pairwise drug similarities for the 982 drugs with known indications by calculating the Tanimoto Coefficient between their interacting proteins profiles and evaluate the prediction performance with "Leave-One-Out" cross-validation.

We observe that using the drug-protein interaction in DMAP, the repurposing performance significantly increases, compared to the performance using the same type of information in CMAP. The Overall AUC for the prediction based on DMAP achieved 0.82. Most importantly, early retrieval performed well, with a partial AUC of 0.72 for a specificity of $90 \%$ or above[26]. Since one could only test the limited number of drugs in experimental setting, the good performance in high specificity region, approximately corresponding to the top ten candidates of all the predictions, would make the proposed drug repositioning more meaningful in practice.

In comparison, we performed similar analysis based on CMAP transcriptome data and the overall AUC was 0.64. The early retrieval performance was only 0.55 . Figure 7 showed that the ROC curve based on DMAP was above the curve from CMAP.

To rule out the possibility that the performance difference was purely due to the drug coverage difference between DMAP and CMAP, we conducted the ROC analysis with only the shared drugs between DMAP and CMAP. The DMAP achieved an AUC of 0.82 while CMAP only achieved an AUC of 0.64 (Figure 8).

Out of all the possible drug pairs, we identified 3,014 significant pairs by requiring the number of overlapped proteins to no less than two and the drug similarity score at the top $5 \%$ of the distribution. The resulting drug network showed a scale-free property (Figure 9), commonly observed in a biological network. Most of the drugs are well connected and formed communities. In fact, 451 drug pairs out of these 3,014 significant pairs have shared at least one known disease indication. For the remaining 2,563 pairs without overlapping indications, the novel drug-disease associations from 1,206 drug pairs were supported by at least one clinical type PubMed article. Table 2 lists the top 20 drug-disease pairs and could be a good starting point for further experimental validations.

\section{DMAP outperforms CMAP in repurposing using} Kolmogorov-Smirnov approach

We compiled the gene expression profiles for 45 distinct diseases and then queried them against DMAP and CMAP, respectively, to generate a ranked list of potential treatments for each of the diseases of interest. By using DMAP drug-protein interaction data, we were able to 


\section{A) GO terms overrepresented in DMAP}

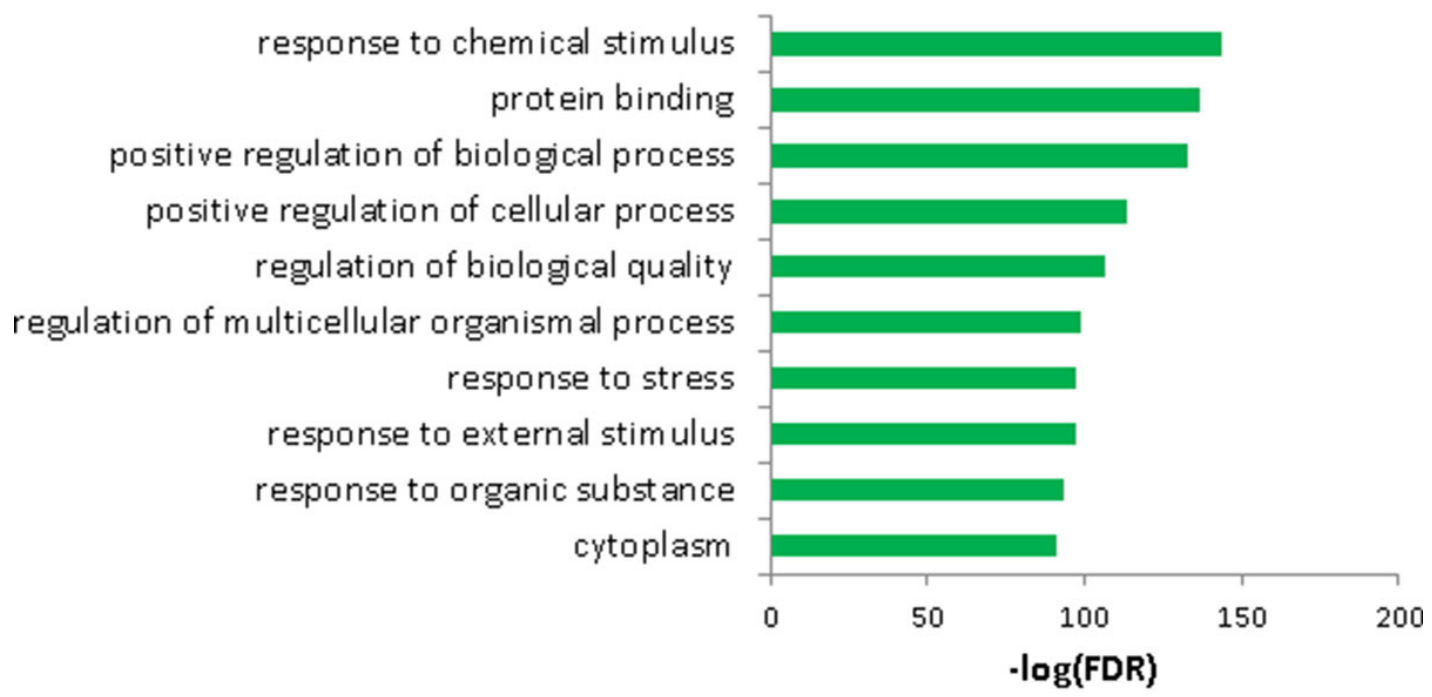

B) GO terms under-represented in DMAP

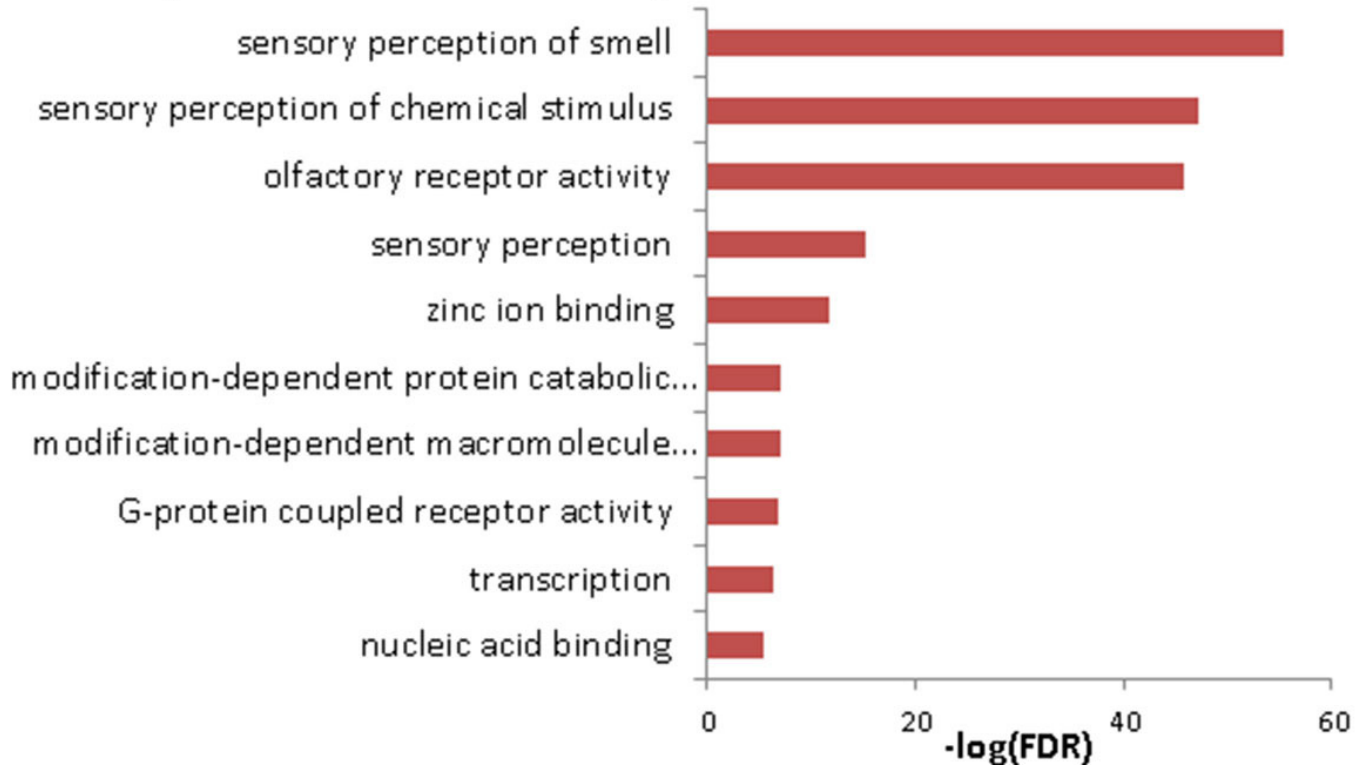

Figure 6 Top 10 gene ontology terms over-represented (A) and under-represented (B) covered by DMAP's proteins. The $\times$ axis is the $-\log ($ FDR $)$ of gene ontology analysis acquired from DAVID functional annotation tool.

correctly retrieve the drugs' indications, which were unable to be retrieved using CMAP drug-protein interaction data. We examined results for diseases that are the leading causes of death in the US [27]. For breast cancer, with the DMAP, we successfully retrieved Anastrozole, Capecitabine, Doxorubicin, Estradiol, Megestrol, Paclitaxel, Testosterone and Testolactone as possible therapeutic drugs for breast cancer. With the CMAP data, only Paclitaxel was retrieved as a potential therapeutic drug. For lung cancer, we retrieved Cisplatin and Etoposide by using the DMAP. However, when CMAP was used, we were not able to retrieve any drugs for lung cancer. Additional file 1 also contains the results for other diseases. To have statistical significance, we required a $p$-value of less than 0.05. CMAP did relatively better in the case for Alzheimer's disease and Leukemia. For these known relationships covered in CMAP but not DMAP, or vice-versa, some were due to having a borderline 


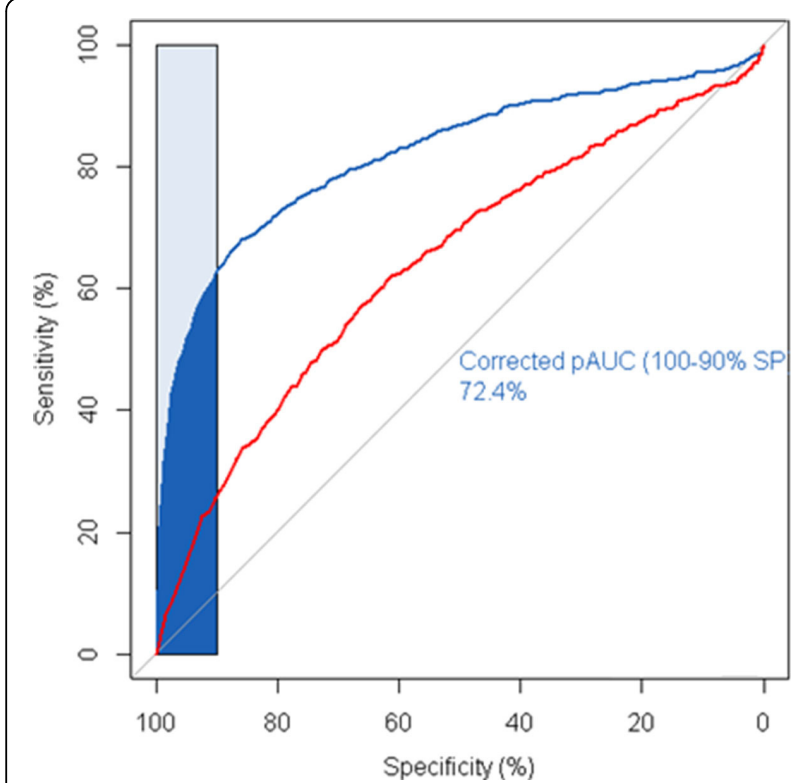

Figure 7 ROC curves for the prediction performance based on DMAP (blue line) and CMAP (red line). Blue shade area provides a partial ROC area corresponding to specificity $90 \%$ above.

$p$-value while others were due to violating our hypothesis of negative correlation. Overall, DMAP and CMAP database were complimentary to each other.

Besides recalling the known drug-disease relationships, with DMAP, the Kolmogorov-Smirnov approach could also propose novel drug-disease associations. National Center for Advancing Translational Sciences (NCATS) [13]

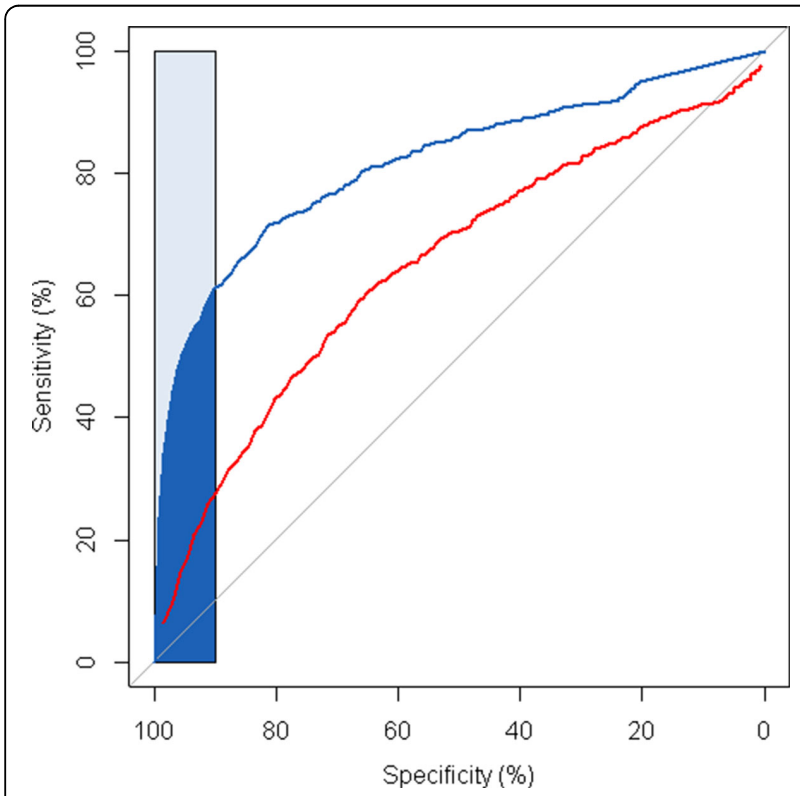

Figure 8 The ROC curves for DMAP and CMAP using the overlapped drugs. provides a list of drugs for translational medical research. We cross checked the novel predictions with their drug list. Here, we highlight three case studies for Vincristine, Nifedipine and Progestrone. Vincristine is a drug typically indicated for Leukemia and Wilm's tumor. A recent study performed by Indolfi et al.[28] revealed that there is a potentially higher rate of survival in patients with bilateral Wilm's tumor when patients are given a dosage of vincristine/actinomycin D. Nifedipine is indicated to treat high blood pressure and angina. The DMAP results suggest that Nifedipine can also be used to treat asthma. Since Nifidipine is a PKC inhibitor and PKC is a potential therapeutic target for asthma [29], it is a potential treatment for asthma. Cheng et al [30] demonstrated in their study that Nifedipine can help control the constriction involved in sensitized tissue in asthma. Furthermore, another study by Barnes et al [31] suggested that Nifidipine modifies exercise-induced asthma. Progesterone is a prescription drug used for women taking estrogens after menopause and is also used for treating amenorrhea. The DMAP results suggest that progesterone can be used to treat breast cancer. In the study by Groshong et al [32], it was determined that treatment with Progesterone can be used to regulate Breast Cancer cell growth.

Additional file 2 summarized all the novel drug repositioning predicted by both similarity approach and KS algorithms, which could be a starting point for further experimental validation.

\section{Conclusions}

Reliable measurements of how drugs affect disease proteins is critical to drug repositioning. In this work we presented a computational drug directionality resource called DMAP to address the challenges. We demonstrated that the resource can greatly facilitate the drug discovery process for the following reasons: access to disease gene-drug relationship data with high coverage and quality; incorporating prior knowledge about biological significance with protein interaction network.

This study differs from previous research in that it provides a comprehensive database of computationally derived drug-protein relationships. Previous efforts $[2,3,9,10]$ on pairing the expression of drugs and diseases mainly rely on experimental connectivity map. For example, Sirota et al.[2] performed a large-scale integration of expression signatures of human diseases from the public data with CMAP drug signatures. This work provides another alternative resource of directed drugprotein relationships. The drug similarity study proves the validity of the probabilistic-based directionality for each drug-protein relationship. The implementation of $\mathrm{K}-\mathrm{S}$ algorithm proves the compatibility of the pharmacology score based ranking with the expression based ranking in CMAP for the drug repositioning research. 

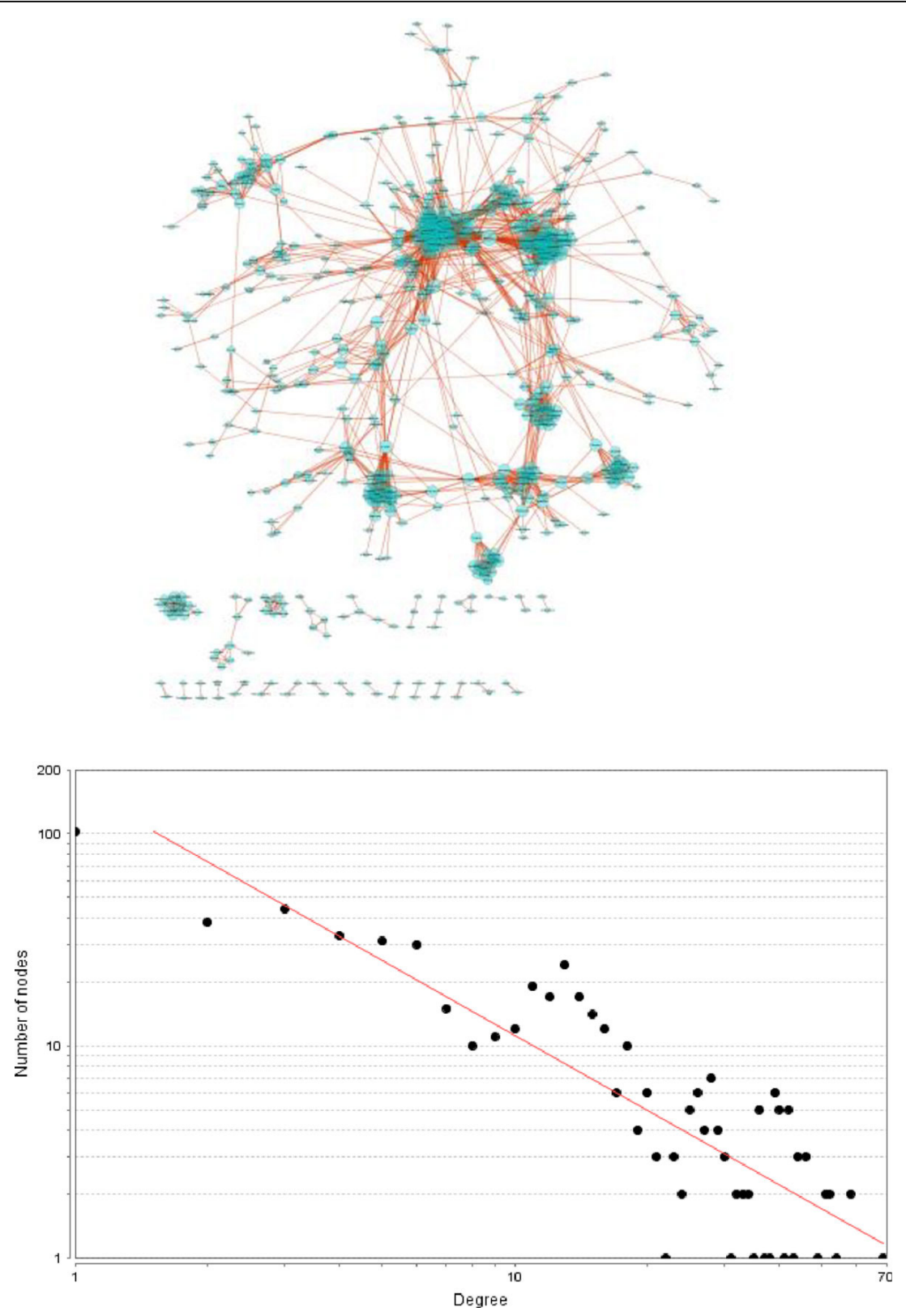

Figure 9 Power-law degree distribution of drug similarity network.

With these two major drug repositioning approaches, the knowledge base from DMAP performed better than directly using the microarray data from CMAP. It can thus serve as a valuable resource for drug repositioning studies.

One limitation of DMAP lies in that the number of interacting proteins for each drug is not a constant number. For the gene expression based profiles in the CMAP database, each drug was measured against the same number of proteins in experiments while in DMAP the number of interacting proteins varies from drug to drug. In DMAP, 13,717 drugs have at least
10 activated and inhibited proteins. Despite this limitation, the database served its purpose for systematic drug repositioning as demonstrated in this work.

Another limitation of DMAP is the dependency of drug-protein interaction scoring on protein-protein interaction (PPI) databases. As mentioned in [33], disease gene ranking should be performed using PPI data not only with reasonable quality but also high data coverage. In this work, we only used the PPI data to calculate the protein weight. Therefore, we believe the conclusions above still hold. In other words, we expect time and PPI quality to affect primarily drug-protein 
Table 2 Top 20 novel drug repositioning candidate identified and the count of PubMed publication support the proposed clinical indications

\begin{tabular}{lll}
\hline Drug & Disease & $\begin{array}{l}\text { PubMed } \\
\text { (Clinical) }\end{array}$ \\
\hline Rocuronium & Pain & 126 \\
\hline Clemastine & Allergies & 80 \\
\hline Mometasone & Asthma & 78 \\
\hline Nicotinamide & Alzheimer Disease & 45 \\
\hline Sotalol & Hypertension & 42 \\
\hline Sertraline & Alzheimer Disease & 40 \\
\hline Ifosfamide & Leukemia, Acute Myeloid & 40 \\
\hline Gabapentin & Anxiety disorder & 33 \\
\hline Vinorelbine & Prostate Cancer & 32 \\
\hline Lumiracoxib & Pain & 28 \\
\hline Hydrocodone & Anesthetic & 25 \\
\hline Zileuton & Inflammatory diseases & 20 \\
\hline Irbesartan & Cardiovascular disease & 17 \\
\hline Moclobemide & Parkinson Disease & 13 \\
\hline Fluvoxamine & Alzheimer Disease & 10 \\
\hline Ranolazine & Dysrhythmias & 6 \\
\hline Trihexyphenidyl & Depression & 5 \\
\hline Nicotinamide & Breast Cancer & 5 \\
\hline Methylphenidate & Obesity & 5 \\
\hline Pemetrexed & Colon cancers & 1 \\
\hline
\end{tabular}

data significantly if and only if the drug-protein relationship score is relatively low; when the drug-protein relationship is high - suggesting that there're lots of data coverage for the relationships across many literature reports - the time or PPI quality effect is expected to be relatively small.

\section{Funding}

Publication of this article was funded in part by the National Institute of Health to Dr Jake Chen (co-PI of R21CA173918).

\section{Additional material}

Additional File 1: Retrieval of known disease drug relationships from DMAP and CMAP, respectively.

Additional File 2: Drug repositioning predicted by both similarity approach and KS algorithms. Note: in both files, the star rating is labelled according to the following criteria:K-S Score<-0.3: ««««-0.3 $\leq$ K-S Score<-0.2: «««-0.2 $\leq$ K-S Score<-0.1: ««แ-0.1 $\leq$ K-S Score<0: ««K-S Score $\geq$ 0 or $p$-value $\geq 0.05:$ «

\section{Competing interests}

The authors declare that they have no competing interests.

\section{Authors' contributions}

$\mathrm{HH}$ performed database construction, drug repositioning performance evaluations and predictions, and led the writing of the manuscript under the guidance of JYC. TN, SI, ZLY and SS participated in data analysis and helped with documentation of the case studies. JYC conceived the project, supervised the entire research team with frequent feedback in the design, implementation, and evaluation of the project, and revision of the manuscript. All authors contributed to the completion of the manuscripts.

\section{Acknowledgements}

We acknowledge the support of Indiana Center for Systems Biology and Personalized Medicine during the design and implementation of the project. Our database servers and web applications are hosted and maintained with the generous support of Indiana University Information Technology and Support group.

This article has been published as part of BMC Bioinformatics Volume 16 Supplement 13, 2015: Proceedings of the 12th Annual MCBIOS Conference. The full contents of the supplement are available online at http://www. biomedcentral.com/bmcbioinformatics/supplements/16/S13.

\section{Authors' details}

'School of Informatics and Computing, Indiana University, Indianapolis, IN 46202, USA. ${ }^{2}$ Department of Computer and Information Science, Purdue University, Indianapolis, IN 46202, USA. ${ }^{3}$ School of Medicine, Indiana University, Indianapolis, IN 46202, USA. Institute of Biopharmaceutical Informatics and Technology, Wenzhou Medical University, Zhejiang, China.

Published: 25 September 2015

\section{References}

1. Gottlieb A, et al: PREDICT: a method for inferring novel drug indications with application to personalized medicine. Mol Syst Biol 2011, 7:496.

2. Sirota $\mathrm{M}$, et al: Discovery and preclinical validation of drug indications using compendia of public gene expression data. Sci Transl Med 2011, 3(96):96ra77.

3. Iorio $F$, et al: Discovery of drug mode of action and drug repositioning from transcriptional responses. Proc Natl Acad Sci USA 2010, 107(33):14621-6.

4. DiMasi JA, RW Hansen, HG Grabowski: The price of innovation: new estimates of drug development costs. J Health Econ 2003, 22(2):151-85.

5. Ashburn TT, KB Thor: Drug repositioning: identifying and developing new uses for existing drugs. Nat Rev Drug Discov 2004, 3(8):673-83.

6. Keiser MJ, et al: Predicting new molecular targets for known drugs. Nature 2009, 462(7270):175-81

7. Campillos $M$, et al: Drug target identification using side-effect similarity. Science 2008, 321(5886):263-6.

8. Li J, X Zhu, JY Chen: Building disease-specific drug-protein connectivity maps from molecular interaction networks and PubMed abstracts. PLoS Comput Biol 2009, 5(7):e1000450.

9. Hu G, P Agarwal: Human disease-drug network based on genomic expression profiles. PLoS One 2009, 4(8):e6536.

10. Lamb J: The Connectivity Map: a new tool for biomedical research. Nat Rev Cancer 2007, 7(1):54-60.

11. Lamb J, et al: The Connectivity Map: using gene-expression signatures to connect small molecules, genes, and disease. Science 2006, 313(5795):1929-35

12. Dickson M, JP Gagnon: The cost of new drug discovery and development. Discov Med 2004, 4(22):172-9.

13. Huang R, et al: The NCGC pharmaceutical collection: a comprehensive resource of clinically approved drugs enabling repurposing and chemical genomics. Sci Transl Med 2011, 3(80):80ps16..

14. Kuhn M, et al: STITCH 3: zooming in on protein-chemical interactions. Nucleic Acids Res 2012, 40(Database):D876-80.

15. Davis AP, et al: The Comparative Toxicogenomics Database's 10th year anniversary: update 2015. Nucleic Acids Res 2015, 43(Database):D914-20.

16. Chen JY, S Mamidipalli, T Huan: HAPPI: an online database of comprehensive human annotated and predicted protein interactions. BMC Genomics 2009, 10(Suppl 1):S16.

17. Chen JY, C Shen, AY Sivachenko: Mining Alzheimer disease relevant proteins from integrated protein interactome data. Pac Symp Biocomput 2006, 367-78. 
18. Zhu F, et al: Update of TTD: Therapeutic Target Database. Nucleic Acids Res 2010, 38(Database):D787-91.

19. Pacini $C$, et al: DvD: An R/Cytoscape pipeline for drug repurposing using public repositories of gene expression data. Bioinformatics 2013 29(1):132-4.

20. Barrett T, et al: NCBI GEO: archive for functional genomics data sets-update. Nucleic Acids Res 2013, 41(D1):D991-5.

21. Irizarry RA, et al: Summaries of Affymetrix GeneChip probe level data. Nucleic Acids Res 2003, 31(4):e15.

22. Warnat $P, R$ Eils, B Brors: Cross-platform analysis of cancer microarray data improves gene expression based classification of phenotypes. BMC Bioinformatics 2005, 6:265.

23. Chiang AP, AJ Butte: Systematic evaluation of drug-disease relationships to identify leads for novel drug uses. Clin Pharmacol Ther 2009, 86(5):507-10.

24. Zhu F, et al: Therapeutic target database update 2012: a resource for facilitating target-oriented drug discovery. Nucleic Acids Res 2012, 40(Database):D1128-36.

25. Huang da W, et al: DAVID Bioinformatics Resources: expanded annotation database and novel algorithms to better extract biology from large gene lists. Nucleic Acids Res 2007, 35(Web Server):W169-75.

26. Walter SD: The partial area under the summary ROC curve. Stat Med 2005, 24(13):2025-40.

27. Hoyert LDonna, Xu Jiaquan: Deaths: Preliminary Data for 2011. National Vital Statistics Reports 2012, 61(6), October 10.

28. Indolfi P, et al: Synchronous bilateral Wilms tumor: A report from the Associazione Italiana Ematologia Oncologia Pediatrica (AIEOP). Cancer 2013

29. Do JS, et al: Therapeutic target validation of protein kinase C(PKC)-zeta for asthma using a mouse model. Int J Mol Med 2009, 23(4):561-6.

30. Cheng $D$, et al: The effects of protein kinase $C$ (PKC) on the tension of normal and passively sensitized human airway smooth muscle and the activity of voltage-dependent delayed rectifier potassium channel (Kv). J Huazhong Univ Sci Technolog Med Sci 2007, 27(2):153-6.

31. Barnes PJ, NM Wilson, MJ Brown: A calcium antagonist, nifedipine, modifies exercise-induced asthma. Thorax 1981, 36(10):726-30.

32. Groshong $\mathrm{SD}$, et al: Biphasic regulation of breast cancer cell growth by progesterone: role of the cyclin-dependent kinase inhibitors, p21 and p27(Kip1). Mol Endocrinol 1997, 11(11):1593-607.

33. Huang $\mathrm{H}, \mathrm{J} \mathrm{Li}$, JY Chen: Disease gene-fishing in molecular interaction networks: a case study in colorectal cancer. Conference proceedings :... Annual International Conference of the IEEE Engineering in Medicine and Biology Society IEEE Engineering in Medicine and Biology Society Conference 2009, 2009:6416-9.

doi:10.1186/1471-2105-16-S13-S4

Cite this article as: Huang et al: DMAP: a connectivity map database to enable identification of novel drug repositioning candidates. BMC

Bioinformatics 2015 16(Suppl 13):S4.

\section{Submit your next manuscript to BioMed Central and take full advantage of:}

- Convenient online submission

- Thorough peer review

- No space constraints or color figure charges

- Immediate publication on acceptance

- Inclusion in PubMed, CAS, Scopus and Google Scholar

- Research which is freely available for redistribution

Submit your manuscript at www.biomedcentral.com/submit 